\title{
Disjoint genus-0 surfaces in extremal graph theory and set theory lead to a novel topological theorem
}

Arturo Tozzi

Center for Nonlinear Science, Department of Physics, University of North Texas, Denton, Texas, USA

* Correspondence: tozziarturo@libero.it; arturo.tozzi@alsnapoli2nord.it

\begin{abstract}
When an edge is removed, a cycle graph $\mathrm{C} n$ becomes a $n-1$ tree graph. This observation from extremal set theory leads us to the realm of set theory, in which a topological manifold of genus- 1 turns out to be of genus- 0 . Starting from these premises, we prove a theorem suggesting that a manifold with disjoint points must be of genus-0, while a manifold of genus-1 cannot encompass disjoint points.
\end{abstract}

KEYWORDS: Combinatorics; Ramsey's theory; Borsuk-Ulam theorem; black hole; singularity.

\section{PREMISES}

In extremal graph theory, one may ask: given an undirected, connected cycle graph $\mathrm{C} n$ of order $n$ (Figure $\mathbf{A}$ ), what is the maximum possible number of edges such that the graph does not contain a cycle? The answer is a tree graph with n-1 vertices (Bollobás 2004), in which two vertices are not joined (Figure B).

Here we show how this observation from extremal graph theory leads to consequences in set theory and topology.

Two sets are disjoint if they have no elements in common, or in other words, if their intersection is an empty set (Cormen et al., 2001). Many topological manifolds contain disjoint subsets such that $\mathrm{a} \cap \mathrm{b}=\varnothing$. To provide an example, the opposite antipodal points on the genus- $0 \mathrm{n}$-sphere described by the BorsukUlam theorem cannot meet, therefore are disjoint (Matoušek 2003; Peters 2016).

Here we provide a novel theorem, colloquially labelled the punctured balloon theorem (PBT), confronting jointness and disjointness in different genus manifolds.

\section{THEOREM}

- An orientable genus-1 surface cannot encompass disjoint points. See Figure $\mathrm{C}$ for a pictorial rendering.

\section{Proof}

- Let's assume that a genus-1 surface contains two (or more) disjoint points, such that $\mathrm{a} \cap \mathrm{b}=\varnothing$ (Figure D).

- When a deformation retraction is performed, it unavoidably happens that two (or more) disjoint points a and b:

a) Approach the hole border.

b) End up closer.

- Because the two (or more) disjoint points a and b cannot meet, they leave an empty region connecting the internal of the hole with the external of the genus-1 surface.

- This would mean that the hole gets lost, being the manifold of genus-0 instead of the genus- 1 advocated in the assumption. 
- This is a contradiction.

- Hence, the assumption must be incorrect.

A

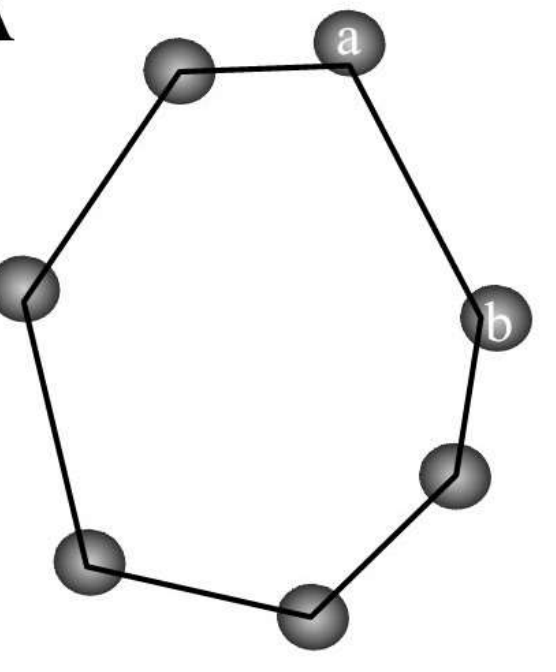

C

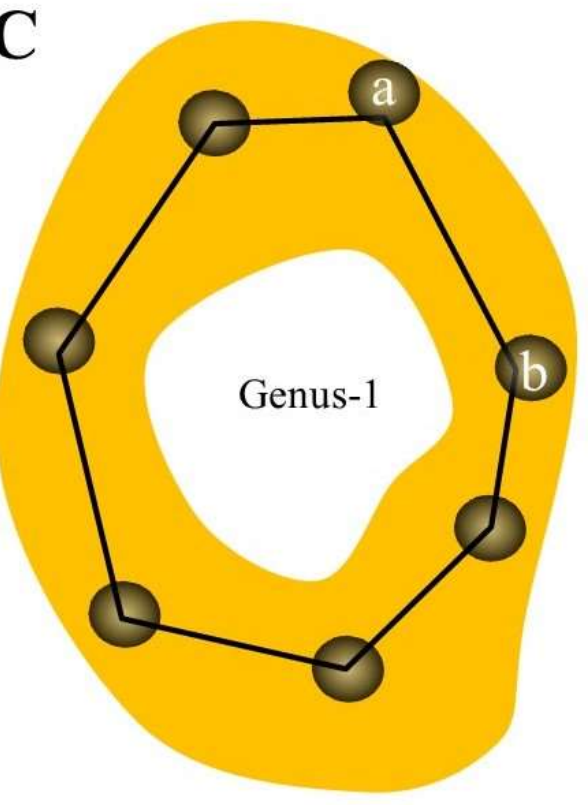

B

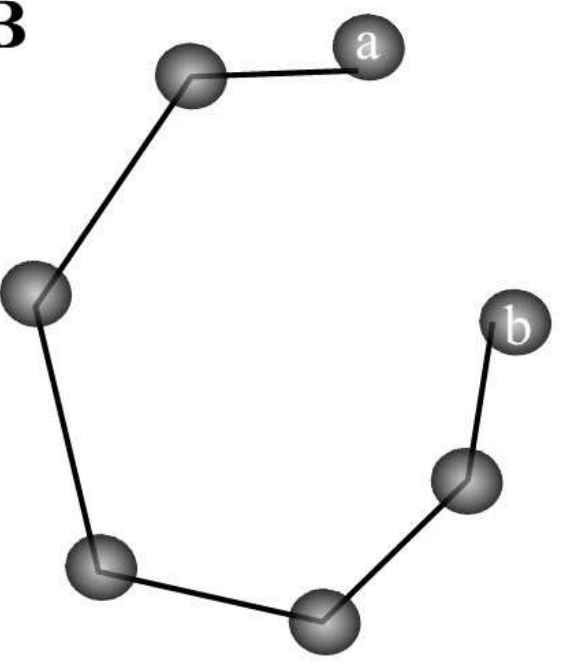

D

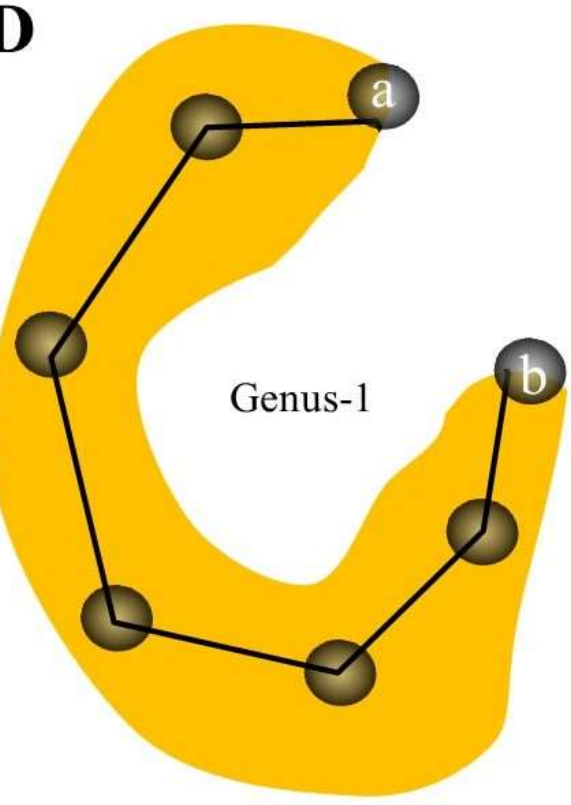

Figure. Relationships among cycle graphs, tree graphs and topological manifold of different genus. Figure A. A cyclic graph. Note that the vertices $a$ and $b$ are joined by an edge. Figure B. When the edge between the vertices a and $b$ is removed, the graph becomes a tree in which the points a and b become the two extremities. Figure C. Topological manifold corresponding to the cyclic graph illustrated in Figure A. Since the manifold cannot contain disjoint points, it turns out to be of genus 1. Figure D. Topological manifold corresponding to the tree graph corresponding to the cyclic graph illustrated in Figure B. Since in this case the two points a and b do not meet, the hole is lost such that the manifold turns out to be of genus 0 .

\section{IMPLICATIONS}

Mathematical outcomes. A manifold including two (or more) disjoint points turns out to be a genus-0 manifold. Note that in our definition of topological manifold we did not use the term "connected". Indeed, PBT casts 
doubts on the role of topological connectedness in the assessment of sets and subsets. Even though deformation retraction is a mapping that captures the idea of continuously shrinking a space into a subspace, PBT suggests something different, i.e., suggests that a genus- 0 manifold must necessarily encompass (at least two) non-intersecting elements. In turn, PBT implies that a genus- 1 surface must contain just intersecting, neighborhood points. When shrinking a genus-1 space into a subspace, BPT entails that all the points gather together, apart from the ones inside the hole. Therefore, a genus- 1 manifold, despite being a set that is not simply connected, must encompass just connected elements, except for the region occupied by the hole.

Physical consequences. PBT rules out the occurrence of continuity/indivisibility among the elements of a physical genus- 0 manifold, holding instead to a discrete operational approach. In touch with this observation, when a choice is performed, e.g., a gauge is introduced in a symmetric manifold (Sengupta et al., 2016), disjoint subsets are generated and manifold connectedness is lost. This means that a genus- 0 manifold, although theoretically shrinkable, must contain some disconnected elements. Take the Poincaré-Brouwer theorem, also termed the "hairy ball" theorem (HBT), stating that there is no non-vanishing continuous tangent vector field on evendimensional n-spheres (Eisenberg and Guy, 1979). This implies that the poles of a genus- 0 sphere are disjoint from the neighbouring elements. PBT suggests that the vanishing tangent vector fields disappear when the ball is drilled completely through: in this case, since the poles have been removed, we achieve a genus-one torus instead of a ball and HBT does not hold anymore.

PBT affects not only disjoint points, but also disjoint features such shapes, functions, vectors, energies, and so on (for a survey, see Tozzi et al., 2017). In physical terms, PBT suggests that a genus-1 manifold must contain homogeneous elements/features/functions all around the hole, since every part of a genus- 1 system is required to topologically intersect with every other part. Therefore, in a physical system encompassing holes - i.e., vortices/antivortices (Padavić et al., 2020), Betti number $\beta_{1} \geq 1$ (Don et al., 2020), topological defects in nanoscopic materials, fragile topology features (Po et al., 2018) and so on - all the regions surrounding the impurities must display at least a few homogeneous elements/features/functions.

This also means that a black hole (Chesler 2019) cannot contain a singularity, unless the black hole itself displays genus $\geq 1$. If a black hole was a genus- 0 manifold (therefore shrinkable to singularity by the huge amount of mass/energy), its own elements would be intersecting. But it runs counter PBT. Hence, the options for a black hole are two:

1) Either the black hole is genus- 0 and singularities are not allowed.

2) Or the black hole is genus $\geq 1$ and its elements can be compressed down to a tiny region surrounding the hole(s).

\section{CONCLUSION}

In this brief note we proposed and demonstrated a novel theorem, based on Ramsey's theory, combinatorics, set theory and topological manifolds. In plain words, take a balloon, that is a genus-one manifold. If you break the jointness by piercing its surface, the hole gest lost and the punctured balloon becomes a genus-0 manifold. Starting from this claim, a topological theorem can be proved plainly stating that "the ends of a donut can meet, whilst the ends of a kidney pie cannot". In the following, we discussed the PBT implications in disparate issues such as topological connectedness, gauge theories and the physics of the black holes. 


\section{REFERENCES}

1) Bollobás B. 2004. Extremal Graph Theory, New York: Dover Publications, ISBN 978-0-486-43596-1.

2) Chesler PM. 2019. Singularities in rotating black holes coupled to a massless scalar field. arXiv:1905.04613.

3) Cormen TH, Leiserson CE, Rivest RL, Stein C. 2001. Data structures for Disjoint Sets. In: Introduction to Algorithms, MIT Press, 498-524. ISBN 0-262-03293-7.

4) Don AP, Peters JF, Ramanna S, Tozzi A. 2020. Topological View of Flows inside the BOLD Spontaneous Activity of the Human Brain. Front. Comput. Neurosci. DOI: 10.3389/fncom.2020.00034.

5) Eisenberg M, Guy R. 1979. A Proof of the Hairy Ball Theorem. The American Mathematical Monthly, 86 (7): $571-$ 574. doi:10.2307/2320587.

6) Matoušek J. 2003. Using the Borsuk-Ulam Theorem. Lectures on Topological Methods in Combinatorics and Geometry. Berlin Heidelberg: Springer-Verlag.

7) Padavić K, Sun K, Lannert K, and Vishveshwara S. 2020. Vortex-antivortex physics in shell-shaped Bose-Einstein condensates. Phys. Rev. A 102, 043305.

8) Peters JF. 2016. Computational Proximity. Excursions in the Topology of Digital Images. Intelligent Systems Reference Library 102. Switzerland: Springer Int. Pub. DOI: 10.1007/978-3-319-30262-1.

9) Po HC, Watanabe H, Vishwanath A. 2018. Fragile Topology and Wannier Obstructions. Phys. Rev. Lett. 121, 126402.

10) Sengupta B, Tozzi A, Coray GK, Douglas PK, Friston KJ. 2016. Towards a Neuronal Gauge Theory. PLOS Biology 14 (3): e1002400. doi:10.1371/journal.pbio.1002400.

11) Tozzi A, Peters JF, Fingelkurts AA, Fingelkurts AA, Marijuán PC. 2017. Topodynamics of metastable brains. Physics of Life Reviews, 21, 1-20. http://dx.doi.org/10.1016/j.plrev.2017.03.001. 